\title{
Origins and Neurochemical Characteristics of Porcine Intervertebral Disc Sympathetic Innervation: a Preliminary Report
}

\author{
Monika Barczewska $^{1}$ • Judyta Juranek ${ }^{2}$ • Joanna Wojtkiewicz ${ }^{2,3,4}$ (D)
}

Received: 28 May 2017 / Accepted: 21 July 2017 /Published online: 31 July 2017

(C) The Author(s) 2017. This article is an open access publication

\begin{abstract}
Intervertebral disc diseases (IVDDs) form a group of a vertebral column disorders affecting a large number of people worldwide. It is estimated that approximately $30 \%$ of individuals at the age of 35 and approximately $90 \%$ of individuals at the age of 60 and above will have some form of disc-affecting pathological changes leading to disc herniation, prolapse and degeneration as well as discogenic pain. Here, we aimed to establish the origins and neurochemical characteristics of porcine intervertebral disc sympathetic innervation involved in pain signalling in IVDD patients. Pigs were given an injection of the Ominipaque contrast agent and Fast Blue (FB) retrograde tracer into the $\mathrm{L}_{4}-\mathrm{L}_{5}$ intervertebral disc and euthanized at 2, 1, and 3 months post injection. Following euthanasia, bilateral sympathetic chain ganglia $(\mathrm{SChG}) \mathrm{Th}_{13}$ to $\mathrm{C}_{1}$ were collected. The presence, distribution and neurochemical characteristics of retrogradely labelled SChG neurons were examined. The majority $(88.8 \%)$ of all $\mathrm{FB}+$ cells were found in the $\mathrm{L}_{3}-\mathrm{L}_{5} \mathrm{SChG}$. Most $\mathrm{FB}+$ neurons stained for dopamine beta hydroxylase (DBH); one-third to one-quarter stained for somatostatin (SOM), neuropeptide Y (NPY) or leu-enkephalin (LENK); and only a few stained for galanin (GAL). Compared with the control, the greatest decline in
\end{abstract}

Joanna Wojtkiewicz

joanna.wojtkiewicz@uwm.edu.pl

1 Department of Neurology and Neurosurgery, Faculty of Medical Sciences, University of Warmia and Mazury, Olsztyn, Poland

2 Department of Pathophysiology, Faculty of Medical Sciences, University of Warmia and Mazury in Olsztyn, Ul. Warszawska 30, 10-082 Olsztyn, Poland

3 Laboratory for Regenerative Medicine, Faculty of Medical Sciences, University of Warmia and Mazury, Olsztyn, Poland

4 Foundation for the Nerve Cells Regeneration, Olsztyn, Poland neurochemical immunostaining was observed 2 weeks post injection, and the lowest decline was noticed 1 month post injection. Our study, for the first time, provides insight into the complex patterns of intervertebral disc sympathetic innervation and suggests that the best time for neurochemical balance restoration therapy would be 1 month post-injury, when the neuronal concentration of all studied substances is close to the initial physiological level, thus providing favourable conditions for successful recovery.

Keywords Intervertebral disc diseases $\cdot$ Retrograde tracer . Pigs $\cdot$ Intervertebral disc sympathetic innervation

\section{Introduction}

Intervertebral disc diseases (IVDDs) are a group of a vertebral column disorders affecting a large number of people worldwide (Teraguchi et al. 2014). It is estimated that approximately $30 \%$ of people at the age of 35 and approximately $90 \%$ of people at the age of 60 and above will have some form of disc-affecting pathological changes leading to disc herniation, disc prolapse and overall disc degeneration, which will, in turn, lead to a wide variety of uncomfortable symptoms, such as a painful degenerating disc or, in severe cases, walking difficulties, thus negatively impacting the quality of life of those affected with the disease (Alshami 2015; Teraguchi et al. 2014).

The available small animal models of intervertebral disc diseases are often inadequate and either fail to provide a complex mechanistic explanation of disc degeneration that is observed in human patients or are fairly limited in uncovering new therapeutic approaches to disc disease prevention and treatment (Alini et al. 2008). To circumvent the limitations of small animal models, in recent years, a great deal of 
attention has been directed towards larger models, such as pigs, which, due to their anatomical and physiological features, more accurately mimic human diseases and reflect the pathological changes underlying many of them in greater detail compared with other animal models. Porcine intervertebral discs are similar in size and structure to human discs, which makes them a very convenient and reliable model for detailed studies of disc deterioration pathogenesis and for testing novel therapeutic approaches to painful disc degeneration in human patients (Busscher et al. 2010).

Sympathetic neurons are thought to contribute to the increased back pain sensation in patients with intervertebral disc degeneration. Studies have shown that so-called "discogenic" pain, which stems from degenerating or injured discs, might be the result of increased sympathetic innervation supplying these discs, contributing to "peripheral sensitization" and increased pain sensation in patients with IVDDs (Peng et al. 2005).

Here, we aim to elucidate the origins and neurochemical characteristics of sympathetic neurons supplying intervertebral discs in large domestic pigs, which are closely physiologically and anatomically similar to humans. The results will provide the groundwork for further preclinical studies of the pathogenesis of disc degeneration and discogenic pain and contribute to clinical experiments on intervertebral disc physiology and pathophysiology in disc disease therapy.

\section{Materials and Methods}

Large White Polish juvenile female pigs, weighing 25 $30 \mathrm{~kg}$, were used in the study. The animals were divided into four groups: one control (C) and three experimental (E1-3). All groups were injected with $350 \mu \mathrm{l}$ of a fluorescent retrograde tracer Fast Blue (FB)/radiologic contrast agent solution (Omnipaque; $300 \mathrm{mg}$ Iohexolum $/ \mathrm{ml}$; Takeda Ireland Ltd., Citywest, Republic of Ireland) into the $\mathrm{L}_{4}-\mathrm{L}_{5}$ intervertebral disc. The duration of the experiment was designed based on previous studies, and the following end time points were established: group E1 - 2 weeks post injection; group E2 - 1 month post injection; and group E3 3 months post injection. In addition to FB injections, the three experimental groups underwent nucleus pulposus dehydration by one-minute disc laser vaporisation (400 J energy pulse, Dornier Medilas D MultiBeam, Germany), followed by introducing a laser fibre optic probe into the intervertebral space with an epidural anaesthesia needle, which was performed under C-arm fluoroscopy (Siemens Medical Solutions) guidance as previously described (Barczewska et al. 2013). The position of the needle was checked radiologically before depositing the solution into the nucleus pulposus of the studied disc. All animal procedures were performed in accordance with Animal Welfare
Guidelines and approved by the local Ethics Committee at the University of Warmia and Mazury, Olsztyn, Poland. All animals were sheltered in the core animal facility at the Faculty of Veterinary Medicine, University of Warmia and Mazury. The care and comfort of each animal was safeguarded in compliance with all pertinent animal welfare laws, regulations, and policies. Each study was planned, conducted and completed with due and acceptable regard for the welfare of the animal.

All animals in one experimental group were euthanized with pentobarbital overdose at their respective end time points and transcardially perfused with a freshly prepared $4 \%$ buffered paraformaldehyde. The bilateral sympathetic chain ganglia $(\mathrm{SChG})$ from $\mathrm{Th}_{15}$ to $\mathrm{S}_{3}$ were collected, cryoprotected and sectioned on a cryostat into $10-\mu \mathrm{m}$ thick serial sections for further analysis. Each section was examined for the presence, distribution and diameter of FBpositive SChG neurons under a BX61 fluorescence microscope (Olympus, Poland). The immunofluorescent characterisation of the $\mathrm{SChG}$ neurochemical phenotype was performed according to the standard protocol as previously described (Barczewska et al. 2013), and the following primary antibodies were used: dopamine beta hydroxylase (DBH), neuropeptide Y (NPY), galanin (GAL), leuenkephalin (LENK) and somatostatin (SOM) (Table 1). The omission or replacement of primary antibodies on sections from each tissue sample set was carried out simultaneously with the experimental staining.

Statistical analysis was performed using GraphPad Instat (GraphPad, La Jolla, CA, USA). All values are presented as the mean \pm standard error (SEM).

\section{Results}

After applying the tracer to the nucleus pulposus of the L4L5 intervertebral disc, FB+ sensory neurons were found in the bilateral $\mathrm{Th}_{16}-\mathrm{S}_{2} \mathrm{SChG}$. Most $\mathrm{FB}+$ cells were found between the $\mathrm{L}_{2}$ and $\mathrm{L}_{6} \mathrm{SChG}$, constituting $94.6 \%$ of all $\mathrm{FB}+$ cells. The relative distribution of FB-labelled neurons in the collected SChG was as follows: $\mathrm{Th}_{16}-0.4 \%, \mathrm{~L}_{1}-$ $0.9 \%, \mathrm{~L}_{2} 4.5 \%, \mathrm{~L}_{3}-11.5 \%, \mathrm{~L}_{4}-63.5 \%, \mathrm{~L}_{5}-11.8 \%, \mathrm{~L}_{6}-$ $3.3 \%, \mathrm{~S}_{1}-0.95 \%$, and $\mathrm{S}_{2}-1.1 \%$ of all labelled neurons. The average number of FB+ cells observed in the injected disc was approximately 842 cells per animal (ranging from 651 to 1148 ).

In all four groups, the majority of all $\mathrm{FB}+$ neurons were positive for DBH. One-third to one-quarter of the FB+ neurons stained for SOM, NPY, or LENK. Finally, only a few of the FB+ neurons stained for GAL (Figs. 1, 2, 3). The total number of FB-positive SChG neurons, their neurochemical characteristics and colocalization patterns are presented in Table 2. 
Table 1 List of primary and secondary antibodies used in the study

\begin{tabular}{|c|c|c|c|c|}
\hline \multicolumn{5}{|c|}{ Primary antibodies } \\
\hline Antibody & Code & Host Species & Dilution & Supplier \\
\hline $\mathrm{D} \beta \mathrm{H}$ & MAB 308 & mouse & $1: 1000$ & Chemicon International Inc., UK; www.chemicon.com \\
\hline NPY & NA 1233 & rabbit & $1: 10,000$ & Biomol Research Laboratories Inc., US; www.biomol.com \\
\hline SOM & $8330-0154$ & rabbit & $1: 10,000$ & Biogenesis Inc., UK; www.biogenesis.co.uk \\
\hline GAL & RIN7153 & rabbit & $1: 10,000$ & Peninsula Labs, US; see Bachem AG; www.bachem.com \\
\hline LENK & EA 1149 & rabbit & $1: 10,000$ & Affinitiy BioReagents Inc., UK; www.bioreagents.com \\
\hline \multicolumn{5}{|c|}{ Secondary antibodies } \\
\hline \multicolumn{3}{|l|}{ Reagent } & Dilution & Supplier \\
\hline \multicolumn{3}{|c|}{ Donkey anti-mouse $\operatorname{IgG}(\mathrm{H}+\mathrm{L})$ FITC conjugated } & $1: 800$ & 715-095-151; Jackson IR Lab, US; www.jacksonimmuno.com \\
\hline \multicolumn{3}{|c|}{ Biotinylated goat anti-rabbit immunoglobulins } & $1: 1000$ & E0432, DAKO Corporation, US, www.dakousa.com \\
\hline \multicolumn{3}{|c|}{$\begin{array}{l}\text { Biotin conjugated } \mathrm{F}(\mathrm{ab})^{\prime} \text { fragment of affinity purified anti-rabbit } \\
\operatorname{IgG}(\mathrm{H}+\mathrm{L})\end{array}$} & $1: 1000$ & 711-1622, BioTrend, Germany; www.biotrend.com \\
\hline \multicolumn{3}{|c|}{ CY3- conjugated Streptavidin } & $1: 9000$ & 016-160-084, Jackson IR Lab, US; www.jacksonimmuno.com \\
\hline
\end{tabular}

In the control group, the highest number of $\mathrm{FB}+$ neurons was positive for DBH $(80 \% \pm 1.5 \%)$ and NPY $(75.6 \% \pm 2.65 \%)$. The number of FB+ labelled for SOM, LENK and GAL was relatively smaller and accounted for $18.6 \% \pm 0.7 \%, 17.8 \pm 1.7 \%$, and $5.5 \% \pm 0.5 \%$ of all FB+ neurons, respectively. Similar expression patterns were also observed in the experimental groups; however, there was a difference between the numbers of neurons stained for a given substance between all experimental groups studied.

In experimental group 1, the following staining patterns were observed: DBH $(68 \% \pm 1.6 \%)$, NPY $(18.7 \% \pm 0.5 \%)$, $\operatorname{SOM}(16.8 \% \pm 1.1 \%)$, LENK $(8.8 \% \pm 0.75 \%)$ and GAL $(1.9 \% \pm 0.3 \%)$. In experimental group 2 , the staining patterns were as follows: DBH $(77.9 \% \pm 3.1 \%)$, NPY $(38.4 \% \pm 2.3 \%), \operatorname{SOM}(29.4 \% \pm 1.3 \%)$, LENK $15.1 \% \pm 2 \%)$ and GAL $(4.4 \% \pm 1 \%)$. Finally, in experimental group 3, the following staining patterns were observed: DBH $(74.2 \% \pm 2.2 \%)$, NPY $(17.2 \% \pm 0.7 \%)$, SOM $(17.7 \% \pm 2.4 \%), \operatorname{LENK}(5.6 \% \pm 0.1 \%)$, and GAL $(3.5 \% \pm 0.6 \%)$. In all experimental groups, the greatest decline in immunohistochemical expression was observed for NPY (Figs. 1, 2, 3).

\section{Discussion}

Currently, studies on intervertebral disc sympathetic innervation are very scarce and are mostly limited to rat and human post-mortem studies. Here, for the first time, we established the origins of sympathetic neurons supplying the lumbar intervertebral discs and demonstrated changes in neuropeptide expression over time.

The results of our studies show that the vast majority of lumbar $\mathrm{L}_{4}-\mathrm{L}_{5}$ intervertebral disc sympathetic innervation at the injection site stemmed from $\mathrm{SChG}$ neurons localised between $\mathrm{L}_{2}$ to $\mathrm{L}_{6}$; however, a small amount of $\mathrm{FB}+$ sympathetic neurons was also found further from the injection site, spreading from $\mathrm{Th}_{6}$ to $\mathrm{S}_{2}$ in all groups of animals.

At the baseline level (control group), DBH, an enzyme catalysing conversion of dopamine into noradrenaline, considered as a classical marker of sympathetic noradrenergic neurons, was the most prevalent molecule studied, observed in $80 \%$ of all intervertebral disc neurons. NPY was the most prevalent neuropeptide and was present in $75.6 \%$ of all neurons. SOM and LENK were present in 18.6 and $17.5 \%$ of all neurons, respectively. Finally, GAL was the least detectable neurochemical and was observed in only $5.5 \%$ of all intervertebral disc neurons.

The levels of all but one of the neurochemicals declined over time. The greatest reduction in expression was observed two weeks and two months post injection (E1 and E3 group), and the lowest reduction (DBH, NPY, LENK, GAL) and a significant increase (SOM) in expression were noticed one month post injection (E2 group), probably marking this time point as the best window for any post-injection manipulation, drug delivery and cell and tissue grafting.

Until now, the details of neurochemical dynamics and their characteristics have not been analysed. Most of the available data refer to the neurochemical properties of intervertebral disc sensory innervation without much insight into its autonomic part. Studies by Fujimoto Aoik et al. showed that the most prevalent neurochemical in sensory neurons supplying the lumbar and cervical intervertebral discs is CGRP, which is also present in some subsets of stellate ganglion sympathetic neurons supplying cervical intervertebral discs. Previous studies on intervertebral disc degeneration models have not addressed the expression of other sympathetic nervous system-related 

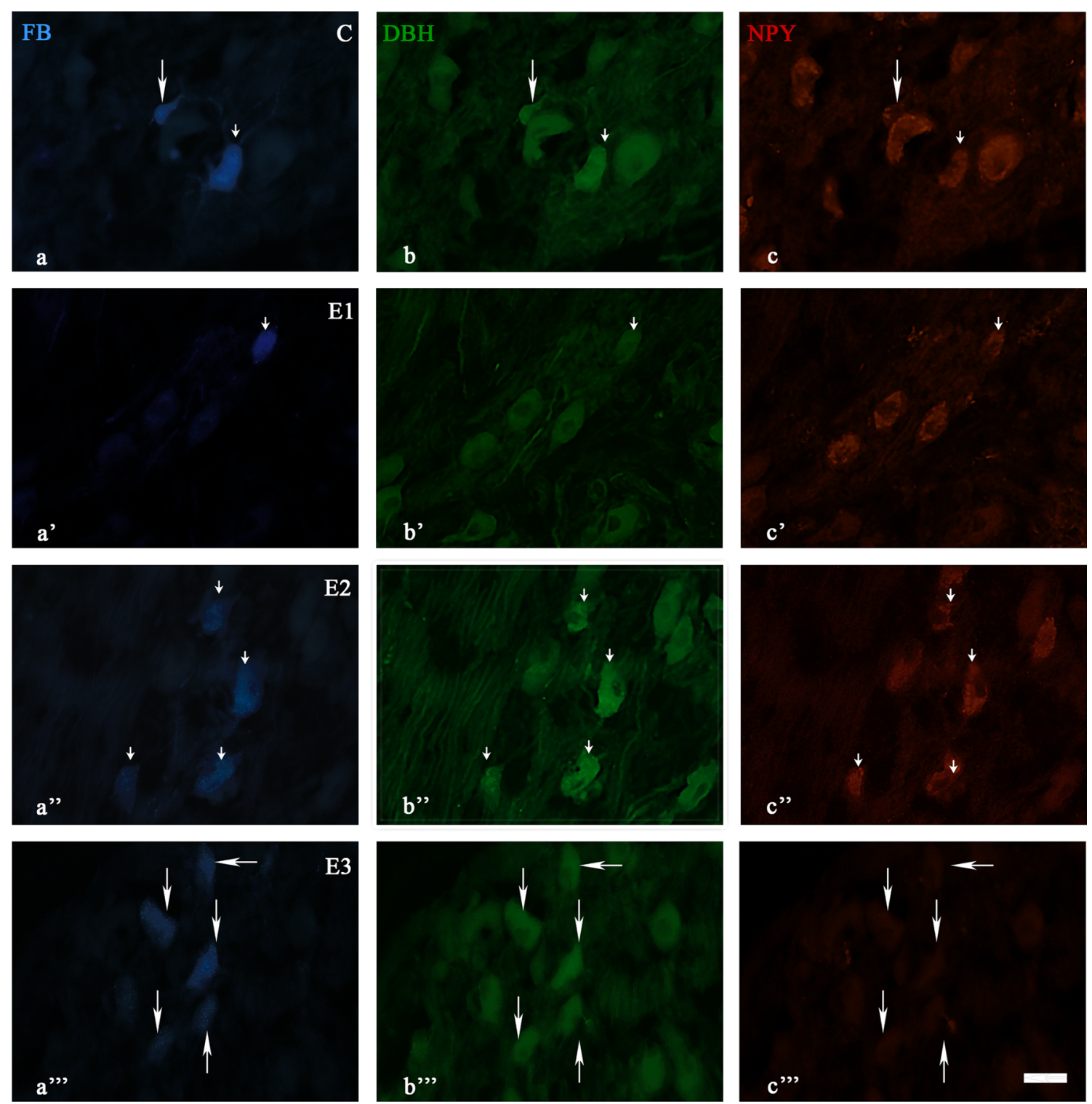

Fig. 1 Immunofluorescent images showing DBH and NPY immunofluorescence in SChG neurons of the control (C) and experimental groups (E1, E2, E3) after FB injection into the porcine intervertebral

discs. The small arrow(s) show(s) the FB-positive neurons containing two examined substances (a-c; a'-c'; a"-c"). The arrow(s) show(s) the FB-positive neuron(s) containing DBH (a-c; a"-c")

molecules, such as DBH, NPY, SOM, LENK, and GAL. Their role in pain transmission in degenerating discs has not been explored in detail; however, recent reports have shown that they are involved in conducting and/or modulating pain that is often manifested in intervertebral disc degeneration as discogenic pain.

The role of the sympathetic noradrenergic innervation in pain transmission and perception has been well established in numerous studies of pain in human patients and animal models (Li et al. 2002; Ma and Eisenach 2003; Wood 2008). The most recent studies by Nascimento et al. have shown that DBH levels are increased in rats after sciatic nerve injury, correlating with mechanical allodynia and pain-like behaviour in these animals. These authors also showed that the chemical suppression of sciatic nerve sympathetic innervation significantly reduced pain perception and decreased sensitivity to cold, thus further supporting the involvement of the noradrenergic system in pain generation (Nascimento et al. 2015).

NPY, which is mainly linked to the regulation of hunger and obesity, has recently been shown to contribute to osteoporosis- and osteoarthritis-related pain generation and perception in postmenopausal women (Xiao et al. 2016), patients with knee osteoarthritis (Wang et al. 2014) and in rat models of this disease (Adaes et al. 2015). Furthermore, studies on NPY expression in animal 

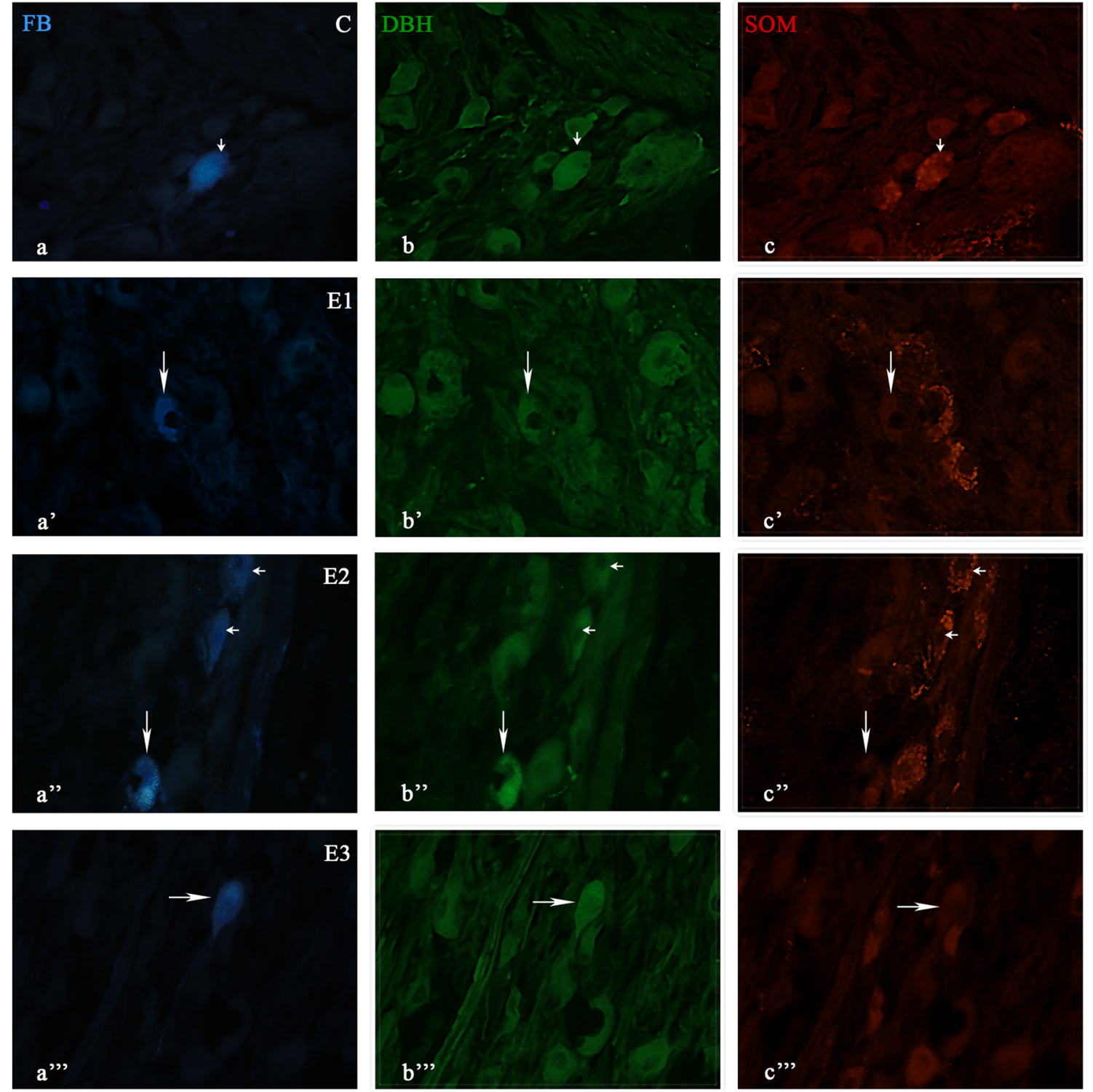

Fig. 2 Immunofluorescent images showing DBH and SOM immunofluorescence in $\mathrm{SChG}$ neurons of the control (C) and experimental groups (E1, E2, E3) after FB injection into the porcine intervertebral

discs. The small arrow(s) show(s) the FB-positive neurons containing two examined substances (a-c; a"-c"). The $\operatorname{arrow}(\mathrm{s})$ show(s) the FBpositive neuron(s) containing DBH (a'-c'; a"-c", a"'-c"')

pain models revealed that NPY was upregulated in the prefrontal cortex and sciatic nerve post-injury, thus further supporting the role of this neuropeptide role in pain generation and perception (Kimura et al. 2015; Magnussen et al. 2015). In addition, recent studies of sensory neurons supplying intervertebral discs in small animal disc degeneration models demonstrated that the expression of NPY is increased, suggesting an enhanced nociceptive response and a contribution to discogenic pain (Miyagi et al. 2014).

SOM is another neuropeptide whose association with pain manifestation has been gradually established over recent years (Kumar 2009). For example, a study by Carlton et al. investigating the role of SOM receptors in analgesia showed that SOM inhibits activity-dependent $\mid$ nociceptors, alleviating pain and reducing pain perception in patients following intrathecal or epidural administration (Carlton et al. 2001). More importantly, SOM has been indicated as a plausible alternative to other dependency-evoking analgesics for alleviating pain during/ post minor surgery procedures and for controlling different forms of pain signals induced by certain conditions and disorders, such as joint inflammation, arthritis, and neuropathy (Abdel-Magid 2015; Carlton et al. 2001; Silveri et al. 1994).

LENK belongs to a well-established family of endogenous opioids, which are naturally occurring painkillers. Recent studies have shown that the stimulation of endogenous LENK production, the administration of exogenous 

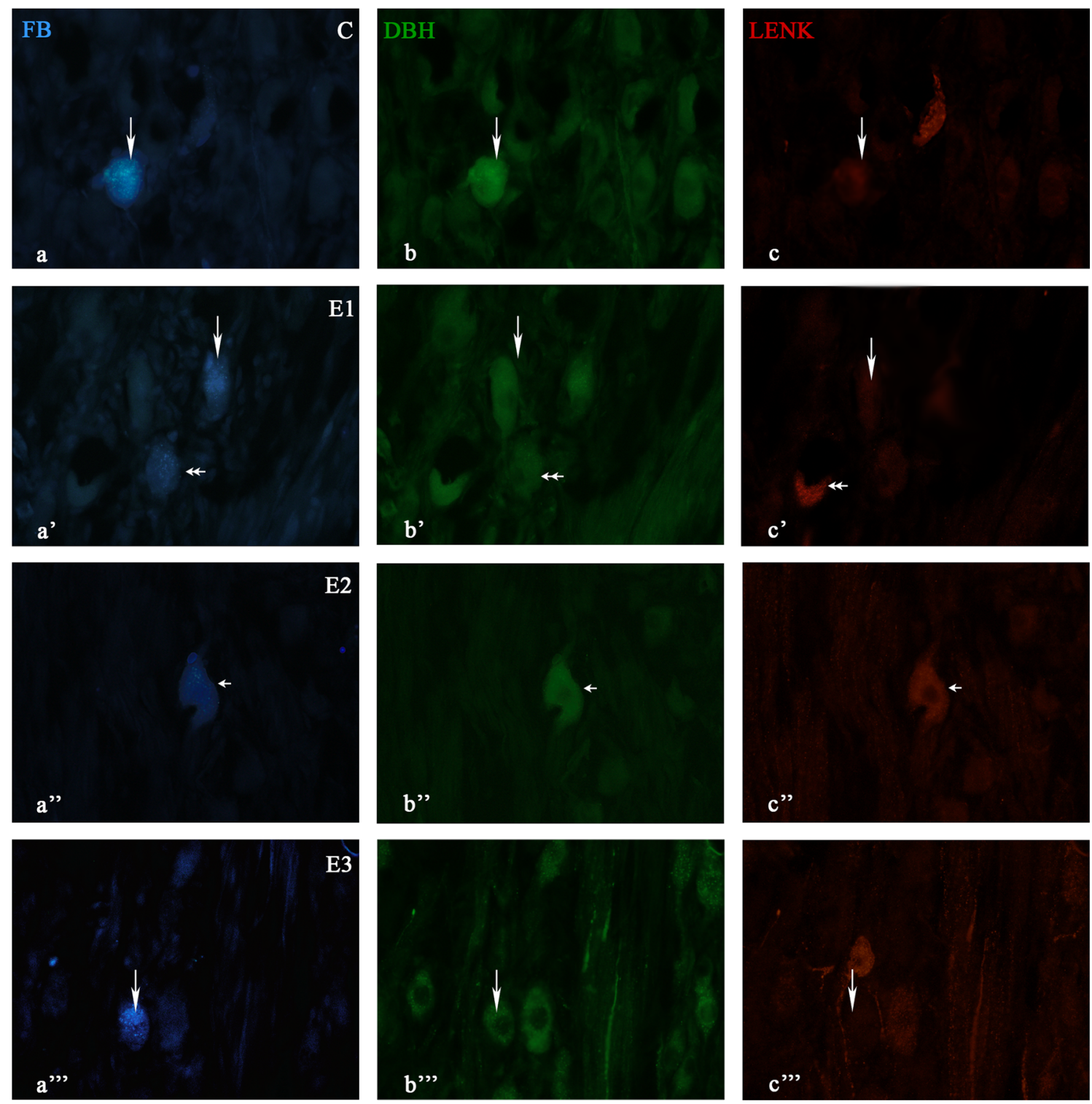

Fig. 3 Immunofluorescent images showing DBH and LENK immunofluorescence in SChG neurons of the control (C) and experimental groups (E1, E2, E3) after FB-injection into the porcine intervertebral discs. The small arrow(s) show(s) the FB-positive neurons containing

two examined substances (a"-c"). The $\operatorname{arrow}(\mathrm{s})$ show(s) the FBpositive neuron(s) containing DBH (a-c; a'-c'; a"'-c"'). The double small arrow indicates LENK (a'-c')

LENK or blocking the activity of endogenous LENKdegrading enzymes affects pain signalling and manifestation by treating chronic pain of various origins (Goins et al. 2012), alleviating visceral pain in mouse models of painful bladder syndrome (Yokoyama et al. 2009), reducing post-injury neuropathic pain in mice (Bonnard et al. 2016), and promoting peripheral opioid analgesia in rats (Akahori et al. 2008; Schreiter et al. 2012). Furthermore, the results of clinical trials on the use of LENK analogues have revealed that the administration of exogenous LENK helps to lessen chronic pain in cancer patients (Moulin et al. 1985), thus underlining the importance of LENK in pain generation and treatment.

Similarly, GAL is yet another example of neuropeptides involved in pain signalling, acting in the suppression of nociceptive receptors and contributing to pain alleviation in animal models of chronic and acute pain (Coronel et al. 2017; Liu and Hokfelt 2002). Extensive studies on the role of GAL in pain transduction and the roles of its agonists and antagonists have been conducted for over 30 years and have presented new evidence for the importance of this neuropeptide in pain modulation and highlighted its role as an effective and less side effectinducing painkiller candidate of the next generation of analgesics used to treat different forms of pain (Lang et al. 2015). It has been shown that the effects of GAL 
Table 2 Neurochemical characteristics of FB-positive neurons supplying porcine intervertebral discs

Immunochemical characteristics

\begin{tabular}{|c|c|c|c|c|c|}
\hline Groups & $\mathrm{FB}+/ \mathrm{DBH}+\mathrm{NPY}+$ & $\mathrm{FB}+/ \mathrm{DBH}+/ \mathrm{NPY}-$ & $\mathrm{FB}+/ \mathrm{DBH}-/ \mathrm{NPY}+$ & $\mathrm{FB}+/ \mathrm{DBH}-/ \mathrm{NPY}-$ & No. of neurons \\
\hline CTRL & $70.1 \pm 2.3$ & $10.3 \pm 0.3$ & $5.5 \pm 3.0$ & $13.7 \pm 3.4$ & 894 \\
\hline E1 & $18.9 \pm 0.7$ & $38.3 \pm 0.6$ & 0 & $42.9 \pm 1.2$ & 789 \\
\hline E2 & $34.6 \pm 3.4$ & $41.2 \pm 6.0$ & 0 & $19.8 \pm 2.5$ & 844 \\
\hline \multirow[t]{2}{*}{ E3 } & $17.6 \pm 1.4$ & $62.5 \pm 1.3$ & 0 & $20.0 \pm 1.8$ & 914 \\
\hline & $\mathrm{FB}+/ \mathrm{DBH}+/ \mathrm{SOM}+$ & $\mathrm{FB}+/ \mathrm{DBH}+/ \mathrm{SOM}-$ & $\mathrm{FB}+/ \mathrm{DBH}-/ \mathrm{SOM}+$ & $\mathrm{FB}+/ \mathrm{DBH}-/ \mathrm{SOM}-$ & \\
\hline CTRL & $17.8 \pm 1.0$ & $64.1 \pm 0.3$ & $1.1 \pm 0.4$ & $16.3 \pm 1.0$ & 1154 \\
\hline E1 & $15.4 \pm 1.8$ & $58.1 \pm 2.5$ & $2.3 \pm 0.2$ & $24.9 \pm 1.4$ & 1017 \\
\hline E2 & $26.1 \pm 2.1$ & $41.4 \pm 2.1$ & $2.9 \pm 1 / 2$ & $29.8 \pm 1.3$ & 919 \\
\hline \multirow[t]{2}{*}{ E3 } & $19.2 \pm 3.1$ & $52.0 \pm 3.5$ & $2.8 \pm 0.3$ & $26.7 \pm 0.4$ & 976 \\
\hline & $\mathrm{FB}+/ \mathrm{DBH}+/ \mathrm{LENK}+$ & $\mathrm{FB}+/ \mathrm{DBH}+/ \mathrm{LENK}-$ & $\mathrm{FB}+/ \mathrm{DBH}-/ \mathrm{LENK}+$ & $\mathrm{FB}+/ \mathrm{DBH}-/ \mathrm{LENK}-$ & \\
\hline CTRL & $15.01 \pm 1.7$ & $66.9 \pm 2.9$ & $3.9 \pm 1.2$ & $14.1 \pm 3.2$ & 862 \\
\hline E1 & $8.8 \pm 1.0$ & $58.9 \pm 2.7$ & 0 & $33.7 \pm 1.8$ & 741 \\
\hline E2 & $14.6 \pm 4.4$ & $70.0 \pm 2.7$ & 0 & $16.1 \pm 2.8$ & 691 \\
\hline \multirow[t]{2}{*}{ E3 } & $4.8 \pm 0.8$ & $69.5 \pm 3.8$ & 0 & $25.8 \pm 2.9$ & 651 \\
\hline & $\mathrm{FB}+/ \mathrm{DBH}+/ \mathrm{GAL}+$ & $\mathrm{FB}+/ \mathrm{DBH}+/ \mathrm{GAL}-$ & $\mathrm{FB}+/ \mathrm{DBH}-/ \mathrm{GAL}+$ & $\mathrm{FB}+/ \mathrm{DBH}-/ \mathrm{GAL}-$ & \\
\hline CTRL & $8.4 \pm 1.3$ & $72.2 \pm 1.3$ & 0 & $19.0 \pm 0.5$ & 816 \\
\hline E1 & $1.8 \pm 0.4$ & $73.5 \pm 0.7$ & 0 & $24.8 \pm 1.0$ & 754 \\
\hline E2 & $0.9 \pm 0.3$ & $74.7 \pm 4.0$ & $4.2 \pm 1.1$ & $17.3 \pm 1.9$ & 761 \\
\hline E3 & $3.2 \pm 1.3$ & $74.6 \pm 0.5$ & 0 & $22.8 \pm 1.6$ & 698 \\
\hline
\end{tabular}

The total numbers of all examined FB-positive SChG neurons and their colocalisation patterns are given. CTRL - control, E1 - experimental group 1 (two weeks post injection), E2 - experimental group 2 (one month post injection), E3 - experimental group 3 (three months post injection). The results are presented as the mean percentage \pm SEM002E

on pain signalling are dose dependent, either alleviating or increasing pain perception in the studied animal pain models (Holmes et al. 2003).

Here, we observed that at the 1-month time point, which was established as a key time point for future experiments, the three studied peptided, i.e., DBH, NPY and GAL, that are directly or indirectly involved in pain generation and its increased manifestation were downregulated in all studied groups. By contrast, the expression levels of SOM and LENK, the two neuropeptides linked to pain suppression and modulation, were increased or remained at a similar level to baseline, respectively. The observed findings indicate that intervertebral disc manipulations trigger detectable changes in the neurochemical complexity of disc sympathetic innervation and likely contribute to altered pain sensation at different time points following clinical intervention. It may be concluded that modulating neuropeptide levels could help to alleviate the pain induced by intervertebral disc degeneration, providing a better outcome for degenerating disc therapy and improving overall prognosis.

It must be noted, however, that our study has certain limitations resulting from its observational nature. Due to ethical and technical considerations, neither actual pain nor the morphology of the injured degenerating disc was measured.
Further studies investigating the complexity of the dynamics of neuropeptides in experimentally induced disc compression and degeneration in pigs have been designed and are likely to provide more answers concerning the role that these neurochemicals play in pain modulation and perception, ultimately contributing to a better understanding of pain signalling in intervertebral disc diseases.

Open Access This article is distributed under the terms of the Creative Commons Attribution 4.0 International License (http:// creativecommons.org/licenses/by/4.0/), which permits unrestricted use, distribution, and reproduction in any medium, provided you give appropriate credit to the original author(s) and the source, provide a link to the Creative Commons license, and indicate if changes were made.

\section{References}

Abdel-Magid AF (2015) Treating pain with somatostatin receptor subtype 4 agonists. ACS Med Chem Lett 6:110-111. doi:10.1021/ ml500538a

Adaes S, Ferreira-Gomes J, Mendonca M, Almeida L, Castro-Lopes JM, Neto FL (2015) Injury of primary afferent neurons may contribute to osteoarthritis induced pain: an experimental study using the collagenase model in rats. Osteoarthr Cartil 23:914-924. doi:10.1016/j. joca.2015.02.010 
Akahori K, Kosaka K, Jin XL, Arai Y, Yoshikawa M, Kobayashi H, Oka $\mathrm{T}$ (2008) Great increase in antinociceptive potency of [Leu5] enkephalin after peptidase inhibition. J Pharmacol Sci 106: 295-300

Alini $\mathrm{M}$ et al (2008) Are animal models useful for studying human disc disorders/degeneration? Eur Spine J 17:2-19. doi:10.1007/s00586007-0414-y

Alshami AM (2015) Prevalence of spinal disorders and their relationships with age and gender. Saudi Med J 36:725-730. doi:10.15537/smj. 2015.6.11095

Barczewska M et al (2013) MR monitoring of minimally invasive delivery of mesenchymal stem cells into the porcine intervertebral disc. PLoS One 8:e74658. doi:10.1371/journal.pone.0074658

Bonnard E, Poras H, Fournie-Zaluski MC, Roques BP (2016) Preventive and alleviative effects of the dual enkephalinase inhibitor (Denki) PL265 in a murine model of neuropathic pain. Eur J Pharmacol 788: 176-182. doi:10.1016/j.ejphar.2016.05.041

Busscher I, Ploegmakers JJ, Verkerke GJ, Veldhuizen AG (2010) Comparative anatomical dimensions of the complete human and porcine spine. Eur Spine J 19:1104-1114. doi:10.1007/s00586010-1326-9

Carlton SM, Du J, Davidson E, Zhou S, Coggeshall RE (2001) Somatostatin receptors on peripheral primary afferent terminals: inhibition of sensitized nociceptors. Pain 90:233-244

Coronel MF, Villar MJ, Brumovsky PR, Gonzalez SL (2017) Spinal neuropeptide expression and neuropathic behavior in the acute and chronic phases after spinal cord injury: effects of progesterone administration. Peptides 88:189-195. doi:10.1016/j.peptides.2017.01. 001

Goins WF, Cohen JB, Glorioso JC (2012) Gene therapy for the treatment of chronic peripheral nervous system pain. Neurobiol Dis 48:255270. doi:10.1016/j.nbd.2012.05.005

Holmes FE et al (2003) Transgenic overexpression of galanin in the dorsal root ganglia modulates pain-related behavior. Proc Natl Acad Sci U S A 100:6180-6185. doi:10.1073/pnas.0937087100

Kimura Y, Ishikawa M, Hori Y, Okabe T, Sakamoto A (2015) Effect of electroconvulsive stimulation on messenger RNA expression in the prefrontal cortex in a rat pain model. Biomed Rep 3:802-806. doi: $10.3892 /$ br. 2015.525

Kumar U (2009) Role of Somatostatin and Somatostatin receptors in pain. In: Peripheral receptor targets for analgesia. John Wiley \& Sons, Inc., Hoboken, New Jersey pp 397-417. doi:10.1002/ 9780470522226.ch16

Lang R, Gundlach AL, Holmes FE, Hobson SA, Wynick D, Hokfelt T, Kofler B (2015) Physiology, signaling, and pharmacology of galanin peptides and receptors: three decades of emerging diversity. Pharmacol Rev 67:118-175. doi:10.1124/pr.112.006536

Li X, Conklin D, Ma W, Zhu X, Eisenach JC (2002) Spinal noradrenergic activation mediates allodynia reduction from an allosteric adenosine modulator in a rat model of neuropathic pain. Pain 97:117-125
Liu HX, Hokfelt T (2002) The participation of galanin in pain processing at the spinal level. Trends Pharmacol Sci 23:468-474

Ma W, Eisenach JC (2003) Chronic constriction injury of sciatic nerve induces the up-regulation of descending inhibitory noradrenergic innervation to the lumbar dorsal horn of mice. Brain Res 970:110-118

Magnussen C, Hung SP, Ribeiro-da-Silva A (2015) Novel expression pattern of neuropeptide $\mathrm{Y}$ immunoreactivity in the peripheral nervous system in a rat model of neuropathic pain. Mol Pain 11:31. doi: 10.1186/s12990-015-0029-y

Miyagi M, Millecamps M, Danco AT, Ohtori S, Takahashi K, Stone LS (2014) ISSLS prize winner: increased innervation and sensory nervous system plasticity in a mouse model of low back pain due to intervertebral disc degeneration. Spine (Phila Pa 1976) 39:13451354. doi:10.1097/brs.0000000000000334

Moulin DE, Max MB, Kaiko RF, Inturrisi CE, Maggard J, Yaksh TL, Foley KM (1985) The analgesic efficacy of intrathecal D-Ala2-DLeu5-enkephalin in cancer patients with chronic pain. Pain 23:213221

Nascimento FP, Magnussen C, Yousefpour N, Ribeiro-da-Silva A (2015) Sympathetic fibre sprouting in the skin contributes to pain-related behaviour in spared nerve injury and cuff models of neuropathic pain. Mol Pain 11:59. doi:10.1186/s12990-015-0062-x

Peng B, Wu W, Hou S, Li P, Zhang C, Yang Y (2005) The pathogenesis of discogenic low back pain. J Bone Joint Surg Br 87:62-67

Schreiter A, Gore C, Labuz D, Fournie-Zaluski MC, Roques BP, Stein C, Machelska H (2012) Pain inhibition by blocking leukocytic and neuronal opioid peptidases in peripheral inflamed tissue. FASEB J 26:5161-5171. doi:10.1096/fj.12-208678

Silveri F, Morosini P, Brecciaroli D, Cervini C (1994) Intra-articular injection of somatostatin in knee osteoarthritis: clinical results and IGF-1 serum levels. Int J Clin Pharmacol Res 14:79-85

Teraguchi $\mathrm{M}$ et al (2014) Prevalence and distribution of intervertebral disc degeneration over the entire spine in a population-based cohort: the Wakayama spine study. Osteoarthr Cartil 22:104-110. doi:10.1016/ j.joca.2013.10.019

Wang L, Zhang L, Pan H, Peng S, Lv M, Lu WW (2014) Levels of neuropeptide $\mathrm{Y}$ in synovial fluid relate to pain in patients with knee osteoarthritis. BMC Musculoskelet Disord 15:319. doi:10.1186/ 1471-2474-15-319

Wood PB (2008) Role of central dopamine in pain and analgesia. Expert Rev Neurother 8:781-797

Xiao J, Yu W, Wang X, Wang B, Chen J, Liu Y, Li Z (2016) Correlation between neuropeptide distribution, cancellous bone microstructure and joint pain in postmenopausal women with osteoarthritis and osteoporosis. Neuropeptides 56:97-104. doi:10.1016/j.npep.2015. 12.006

Yokoyama $\mathrm{H}$ et al (2009) Gene therapy for bladder overactivity and nociception with herpes simplex virus vectors expressing preproenkephalin. Hum Gene Ther 20:63-71. doi:10.1089/hum. 2008.094 